Editorial

\title{
Nosocomial Infections As A Preventable Burden For Health Care Delivery \\ JC Saha
}

Nosocomial infections are a growing issue of preventable disease burden in respect of morbidity and mortality in all countries. Particularly in developing countries it poses an enormous negative impact on health delivery costs. The cost may direct (the cost of medicines, bed occupation etc) or indirect (as patients are deprived of work). Nosocomial infections are defined as infections acquired during hospital care which is not present or incubating at admission. Infections occurring more than 48 hours after admission are usually nosocomial. Definitions to identify nosocomial infections have been developed for specific infection sites (e.g. urinary, pulmonary). These are derived from those published by the Centers for Diseases Control and Prevention (CDC) in the United States of America ${ }^{1,2}$. The most important infections are urinary tract infections, pneumonia, diarrhoea, infections following surgery or invasive medical procedure, maternal and newborn infections.

Nosocomial infections are a significant problem throughout the world and are increasing ${ }^{3}$. For example, nosocomial infection rates range from as low as $1 \%$ in a few countries in Europe and the Americas to more then $40 \%$ in parts of Asia, Latin America and subSaharan Africa ${ }^{4}$. In 1987, a prevalence survey involving 55 hospitals in 14 developing countries in four WHO Regions (Europe, Eastern Mediterranean, South-East Asia and Western Pacific) found an average of $8.7 \%$ of all hospital patients had nosocomial infections. Thus at any time, over 1.4 million patients worldwide will have infectious complications acquired in the hospital ${ }^{5}$. In this survey the highest frequencies were reported from hospitals in the Eastern Mediterranean and South-East Asia Regions, 11.8\% and $10 \%$ respectively ${ }^{6}$. These rates most likely do not reflect the current situation because at that time the HIV/AIDS pandemic was just beginning.

Moreover, the survey did not include any countries in Africa where nosocomial infection rates are much higher. They do, however, provide some guidance as to which types of nosocomial infections occur most frequently in developing countries.

Dr. Jitesh Chandra Saha, FCPS (Surgery), FRCS (Edin) Assistant Professor. Dept. of Surgery, FMC, Faridpur Phone: +88-01711431902. E-mail: Jitesh.saha@yahoo.com
Surgical site infections, urinary tract infections and lower respiratory (pneumonia) infections were the leading types reported. This sequence differs somewhat from what is reported in the US, for example, where urinary and respiratory tract infections are the most common followed by surgical site infections ${ }^{7}$.

\section{Frequency}

Rates of nosocomial infections are markedly higher in many developing countries, especially for infections that are largely preventable (e.g., those following surgical procedures such as cesarean section). In these countries, nosocomial infection rates are high because of a lack of supervision, poor infection prevention practices, and inappropriate use of limited resources and overcrowding of hospitals. Key contributing factors are: increasing use of invasive medical devices (e.g., mechanical ventilators, urinary catheters and central intravenous lines) without proper training or laboratory support; inadequate standards and practices for operating blood transfusion services; use of contaminated intravenous fluids, especially in hospitals making their own IV solutions; antibiotic resistance due to overuse of broad spectrum antibiotics; and unsafe and frequently unnecessary injections. More than $50 \%$ of injections in developing countries are unsafe (i.e., the needle, syringe or both are reused) and many injections are unnecessary (e.g., routine injections of vitamin B-12 or antibiotics $)^{8}$. A major consequence of this is that an estimated 80,000 to 160,000 new HIV infections occur annually in subSaharan Africa, and even more cases of HBV and HCV occur worldwide each year as a result of unsafe injections ${ }^{9}$.

The WHO study and others also found that the highest prevalence of nosocomial infections occurs in intensive care units and acute care surgical and orthopedic wards. Not surprisingly, infection rates are higher among patients with increased susceptibility because of old age and the severity of the underlying disease. To this list should now be added those hospitalized patients with decreased immunity due to AIDS and/or multidrug-resistant tuberculosis. The organisms causing most nosocomial infections usually come from the patient's own body (endogenous flora). They also can come from contact with staff (cross-contamination), contaminated instruments and needles, and the environment (exogenous flora). 


\section{Consequences of nosocomial infection}

Nosocomial infections add to functional disability, emotional stress and may, in some cases, lead to disabling conditions that reduce the quality of life. In addition, nosocomial infections have now become one of the leading causes of death ${ }^{10}$. The impact of nosocomial infections takes on even more significance in resource-poor countries, especially those affected most by HIV/AIDS, because recent findings strongly suggest that unsafe medical care may be an important factor in transmitting $\mathrm{HIV}^{11}$.

During the past 10-20 years little progress has been made in addressing the basic problems responsible for the increasing rates of nosocomial infections in many countries, and in some countries, conditions are actually worsening. Nosocomial infections increase the cost of healthcare in the countries least able to afford them through increased length of hospitalization; treatment with expensive medications (e.g., costly antibiotics and antiretroviral drugs for HIV/AIDS); and use of other services (e.g., laboratory tests, X-rays and transfusions). As a consequence, in resource poor countries, efforts to prevent nosocomial infections must assume even greater importance if progress is to be made in improving the quality of patient care in hospitals and other healthcare facilities.

\section{Prevention}

Most of these infections can be prevented with readily available, relatively inexpensive strategies by: adhering to recommended infection prevention practices, especially hand hygiene and wearing gloves; paying attention to well-established processes for decontamination and cleaning of soiled instruments and other items, followed by either sterilization or highlevel disinfection; and improving safety in operating rooms and other high-risk areas where the most serious and frequent injuries and exposures to infectious agents occur. Hospital authority should take the initiative by forming infection control team which continuously monitors the prevalence and incidence of such infections. State authority should take the responsibility of increasing awareness among all levels of health personnel.

Unfortunately, not all nosocomial infections are preventable. For example, some reflect the influence of advanced age, chronic diseases such as uncontrolled diabetes, end-stage kidney disease or advanced pulmonary emphysema, severe malnutrition, treatment with certain drugs (e.g., antimicrobials, corticosteroids and other agents that decrease immunity), the increasing impact of AIDS (e.g., opportunistic infections) and irradiation.

\section{Reference}

1. Garner JS. CDC definitions for nosocomial infections. Am J Infect Control 1988;16:128-140.

2. Horan TC. CDC definition of nosocomial surgical site infections: a modification of CDC definition of surgical wound infection. Am J Infect Control 1992;13:606-608.

3. Alvarado CJ. The Science of Hand Hygiene: A Self-Study Monograph. University of Wisconsin Medical School and SciHealth Communications 2000.

4. Lynch P. Infection Prevention with Limited Resources. ETNA Communications: Chicago 1997.

5. Tikhomirov E. WHO programme for the control of hospital infections. Chemiotherapia 1987;6(3):148-151.

6. Mayon-White RT. An international survey of the prevalence of hospital-acquired infection. J Hosp Infect 1988;11(Suppl A):43-48.

7. Emori TG, Gaynes RP. An overview of nosocomial infections, including the role of the clinical laboratory. Clin Microbiol Rev $1993 ; 6(4): 428-442$.

8. Simonsen L. Unsafe injections in the developing world and transmission of blood borne pathogens: A review. Bull World Health Organ 1999;77(10):789-800.

9. Kane A. Transmission of hepatitis B, hepatitis C and human immunodeficiency viruses through unsafe injections in the developing world: Model-based regional estimates. Bull World Health Organ 1999;77(10):801-807.

10. Ponce-de-Leon S. The needs of developing countries and the resources required. J Hosp Infect 1991;18 (Suppl A):376-381.

11. Gisselquist D. HIV infections in sub-Saharan Africa not explained by sexual or vertical transmission. Int $J$ STD AIDS 2002;13(10):657-666. 